\title{
EL DIAGRAMA CAUSA - EFECTO COMO APORTE AL TRABAJO COLABORATIVO EN LA GENERACIÓN DE COMPETENCIAS INVESTIGATIVAS EN AMBIENTES VIRTUALES DE APRENDIZAJE
}

\author{
Hermes Mosquera Angulo ${ }^{31}$
}

RESUMEN

El presente artículo refleja los avances logrados en desarrollo del proyecto "Formación de competencias investigativas mediante el trabajo colaborativo en ambientes virtuales de aprendizaje", basado en el avance tecnológico investigativo diagrama causa-efecto como herramienta de uso tecnológico para fomentar las Tecnologías de la Información y la comunicación. Dicho proyecto se adelanta al interior de la Universidad Nacional Abierta y a Distancia - UNAD y en este primer avance pretende conocer en detalle la aceptabilidad del software desde la parte funcional, amigabilidad y facilidad de uso, tomando como referente el curso académico Metodología de la investigación.

De igual manera, se incluyen algunas experiencias que se han evidenciado por parte de los estudiantes objeto de investigación y un análisis de los avances obtenidos en el proyecto que se inicia con la revisión documental y software existente en cada uno de los cursos de formación investigativa, evidenciada a través de las guías académicas y los módulos que acompañan cada uno de los cursos objeto de estudio, así como también algunas publicaciones investigativas existentes en torno a las competencias investigativas especialmente en ambientes académicos con el uso de las Tecnologías de la Información y la Comunicación.

Al finalizar la investigación se pretende medir las competencias investigativas generadas haciendo uso del diagrama causa-efecto como herramienta de trabajo colaborativo en ambientes virtuales de aprendizaje.

Palabras clave: formación investigativa, competencias, desarrollo tecnológico, trabajo colaborativo, diagrama causa-efecto.

\begin{abstract}
This article reflects the progress made in developing the project "Training of investigative skills through collaborative work in virtual learning environments." Based on investigative technological development cause-effect diagram as a tool of use of technology to promote information technology and communication. Project being carried out within the National Open University and Distance "UNAD, in this first step aims to determine in detail the

31 Colombiano. Tutor tiempo completo UnAD, CEAD Popayán. Investigador Grupo Wiki-Wiki del mismo centro. Con estudios de Ingeniería de Sistemas, Especialización en Pedagogía para el desarrollo del Aprendizaje Autónomo. Certificado como Director virtual de curso académico unAD, E-mail: Hermes.mosquera@unad.edu.co
\end{abstract}


acceptability of the software from the functional and user-friendliness, taking as reference the academic Research Methodology.

Besides, it includes some experiences that have been shown by the students under investigation and an analysis of the progress achieved in the project that begins with the review of documents and software on each of the research training courses, as evidenced through academic guides and modules that accompany each of the courses under study, as well as some existing research publication about investigative skills especially in academic settings with the use of Information Technology and Communication.

At the end of the research aims to measure the investigative powers generated by making use of cause-effect diagram as a tool for collaborative work in virtual learning environments.

Key words: research training, skills, technological development, collaborative work, causeeffect diagram.

\section{INTRODUCCIÓN}

La Universidad Nacional Abierta y a Distancia viene desarrollando un programa de formación de formadores ${ }^{32}$ a través de diferentes diplomaturas, como medio de cualificación del personal académico. Siendo coherente con su Misión, es pertinente el presente proyecto de investigación para determinar las competencias investigativas por medio del trabajo colaborativo en ambientes virtuales de aprendizaje a través del diagrama-causa.

El curso Metodología de la investigación es el escenario propicio para motivar al estudiante a que haga parte de un semillero de investigación, dado que la investigación formativa nace desde los pequeños grupos de apoyo que se reúnen con el ánimo de explorar una temática de su interés o profundizar en un tema desconocido.

Dentro de las publicaciones encontradas que tratan el tema hago referencia a la investigación realizada por el grupo de Investigación Gestión Vital, Maldonado, L y otros (2007). ${ }^{33}$ Quienes para abordar el tema de competencias investigativas citan el concepto de competencia, entendido este, como el conjunto de conocimientos, habilidades y actitudes que se aplican en el desempeño de una función productiva o académica; asimismo, se entiende por competencia vista desde un ámbito global el saber hacer en un contexto determinado.

32 http://formacion.unadvirtual.org/moodle/

33 Visibilidad y formación en investigación Maldonado, Landazabal, Hernández, Ruíz, Claro, Vanegas y Cruz. 
Por su parte, Rodríguez Mario y Mena García (2000) en su artículo "Formación reflexiva creativa de competencias Investigativas en los docentes". ${ }^{34}$ El maestro debe prepararse para investigar su realidad como parte del desempeño profesional. Su función investigativa está llamada a convertirse en una de sus herramientas básicas para alcanzar éxito en su labor educativa.

Compartiendo el pensamiento de Escobedo (2001); la presente investigación va encaminada a generar competencias investigativas partiendo de los grupos colaborativos para compartir y aportar desde experiencias individuales con el diagrama causa-efecto; una aplicación web en línea tipo software, diseñada para la construcción colectiva de conocimiento, donde los estudiantes puedan aportar sus ideas y estas sean conocidas por cada uno de los integrantes del grupo. En la investigación se identifican cuatro variables objeto de estudio: la recolección de la información, su análisis, la toma de decisiones y el trabajo colaborativo.

\section{OBJETIVOS}

\section{General}

Fortalecer escenarios de generación de competencias investigativas mediante el uso del diagrama causa-efecto como instrumento de trabajo colaborativo en ambientes virtuales de aprendizaje.

\section{Específicos}

Analizar la documentación actual de de los diferentes cursos que hacen parte del área de formación investigativa al interior de la UNAD.

Desarrollar un aplicativo Causa-efecto, material educativo computarizado con innovación pedagógica y tecnológica como instrumento de trabajo colaborativo para ambientes virtuales de aprendizaje.

Desarrollar escenarios de aprendizaje colaborativo que enriquezcan la formación de competencias en el área investigativa, bajo el uso del diagrama causa-efecto.

\section{METODOLOGÍA}

El proyecto se define como una investigación aplicada, toda vez que busca innovar un proceso de educación con una herramienta tecnológica que impacte el modelo de formación de competencias investigativas; cabe recordar que la investigación aplicada exige una rigurosidad en términos de cuantificación y cualificación del impacto obtenido por la modificación introducida en el proyecto. En este sentido, se trabaja de manera conjunta con tutores de las aulas virtuales de formación investigativa de la UNAD. Se hace uso de la encuesta y la observación directa como medios para la recopilación de información de interés y

34 Publicado en Página Web de CLACSO http:// www.clacso.org. Buenos Aires, abril 2000. 
el análisis de los escenarios actuales de formación de competencias investigativas que en este tema se hayan generado.

\section{Aproximación metodológica}

Para dar respuesta a la pregunta de investigación planteada, una de las actividades iniciales se centró en el análisis metódico del material de base que es utilizado para la formación investigativa, especialmente en los cursos que son comunes para todos los programas formativos. Se tuvieron en cuenta seis cursos comunes de formación investigativa y se exploró el tipo y cantidad de herramientas de software que son recomendadas al interior de su material de estudio. La selección de cursos a analizar se hizo de acuerdo con la naturaleza del mismo en cuanto al nivel de oferta e intencionalidad formativa básica en los procesos investigativos.

Tabla 1. Cursos seleccionados objeto de análisis

\begin{tabular}{|l|c|c|}
\hline \multirow{2}{*}{\multicolumn{1}{|c|}{ Cursos analizados }} & \multicolumn{2}{c|}{ Recomiendan SW } \\
\cline { 2 - 3 } & Técnico & Conceptual \\
\hline 100103 - Metodología de la investigación & 30 & 2 \\
\hline 401533 - Investigación cuantitativa & 0 & 1 \\
\hline 401526 - Paradigmas de la investigación & 0 & 1 \\
\hline 401408 - Investigación educativa & 0 & 2 \\
\hline 207012 - Epistemología del diseño investigativo & 0 & 0 \\
\hline 207030 - Investigación en entornos virtuales & 0 & 0 \\
\hline
\end{tabular}

Una revisión especificada de las herramientas de software que son recomendadas como apoyo a la formación investigativa al interior de los cursos, permite observar que su funcionalidad es bastante detallada y en principio facilitaría la generación de competencias en esta área formativa particular. Es así como se encuentra desde software para la investigación cuantitativa hasta herramientas para la generación de teorías o redes.

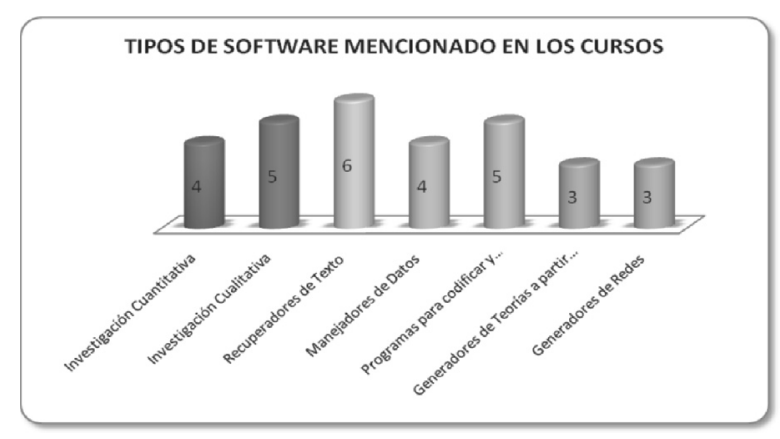

Figura 1. Tipos de Software mencionado en los cursos 
A continuación se especifica el listado de herramientas de software de acuerdo con su naturaleza de utilización mencionado en el material analizado.

\section{AVANCES PARCIALES DE LA INVESTIGACIÓN}

\section{Avance en desarrollo tecnológico}

De forma paralela al desarrollo de la revisión técnica del material didáctico que sirve como base para el estudio de temáticas de formación investigativa, el Grupo de Investigación Wiki-Wiki adelantó, junto con un grupo de estudiantes del Semillero de Investigación en Ambientes Virtuales de Aprendizaje - SIAVA, ${ }^{35}$ la segunda fase de la construcción del software diagrama Causa-Efecto que sea aprovechado como instrumento de mediación pedagógica en la generación de competencias investigativas, especialmente el trabajo en equipo, la recolección de información, el análisis de la información y la toma de decisiones, como variables objeto de estudio.

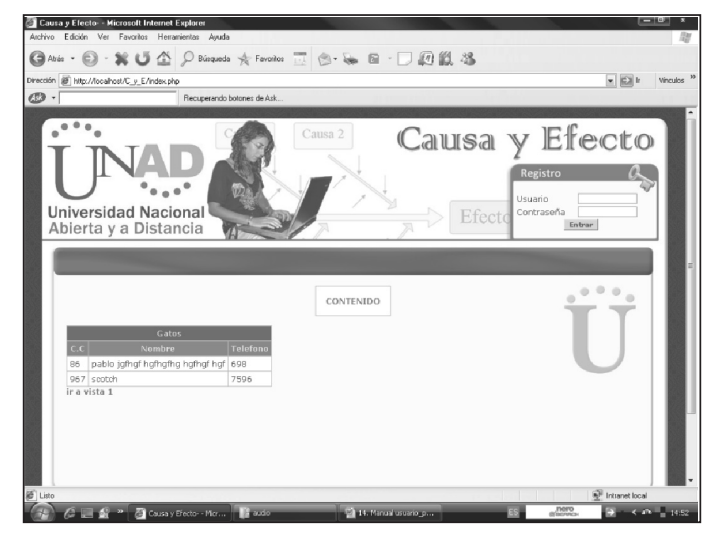

Figura 2. Interfaz inicial del software Causa-Efecto

El entorno de trabajo del software permite la interacción a través de internet mediante una interfaz en la cual los usuarios del mismo (estudiantes y tutores de cursos de formación investigativa) desarrollan actividades colaborativas acorde con los trabajos de campo dentro del desarrollo de una investigación. Puntualmente, el software busca que los estudiantes obtengan una herramienta de análisis de las causas que generan el fenómeno (problema) objeto de investigación, lo representen mediante un diagrama de causas y efecto mientras que soportan todo el proceso a través de herramientas de construcción colaborativa de textos (wiki). Este desarrollo tecnológico (fue validado inicialmente con el grupo de control que actualmente trabaja dentro de esta misma fase del proyecto investigativo), dentro del ciclo de vida se encuentra al $100 \%$ de su construcción.

35 SIAVA Semillero de Investigación en Ambientes Virtuales de Aprendizaje, conformado por estudiantes del programa Ingeniería de sistemas del CEAD de Popayán. Liderado y asesorado por los investigadores del Grupo de Investigación Wiki-Wiki. 


\section{Pruebas de control para competencias específicas}

Se cuenta con un grupo heterogéneo de veinte (20) estudiantes de distintos programas formativos que cursan Metodología de la Investigación y Seminario de Investigación, llamados grupo de control, de los cuales ocho (8) fueron seleccionados para hacer las pruebas iniciales del uso de la herramienta de software. Este grupo de control se ha dividido en dos subgrupos, para el desarrollo investigativo que de solución a una problemática puesta. El equipo de investigador actúa como asesor de la actividad. La información obtenida en el análisis se llevó a cabo a través de la encuesta como instrumento de recolección de datos.

Tabla 2. Resultado individual de la encuesta, pregunta 1

\begin{tabular}{|l|c|c|c|}
\hline \multicolumn{1}{|c|}{ Pregunta } & \multicolumn{2}{|c|}{ Respuestas } & Encuestados \\
\hline $\begin{array}{l}\text { ¿Cree Usted que el uso de la TIC's }{ }^{19} \\
\text { contribuye a la formación integral e } \\
\text { investigativa del estudiante? }\end{array}$ & 17 & No & \\
\hline
\end{tabular}

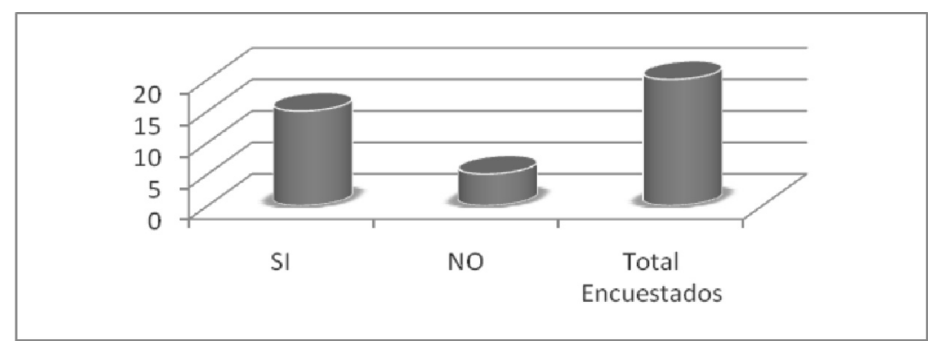

Figura 3. Resultado individual de la encuesta pregunta 1

Los resultados obtenidos en la pregunta 1 , indican que el $85 \%$ de los estudiantes encuestados consideran que el uso de las tecnologías de la información y la comunicación contribuye a la formación investigativa del estudiante. Es coherente con el crecimiento y fortalezas que se vienen presentando con la revolución educativa apoyada por las herramientas informáticas y telemáticas que fortalecen la comunicación, en especial el uso de internet como medio de interacción en la educación en línea e-Liaoning.

Tabla 3. Resultado individual de la encuesta, pregunta 2

\begin{tabular}{|c|c|c|c|}
\hline Pregunta & \multicolumn{2}{|c|}{ Respuestas } & Encuestados \\
\hline $\begin{array}{l}\text { ¿Durante su proceso formativo ha tenido } \\
\text { la oportunidad de realizar trabajos } \\
\text { colaborativos en ambiente virtual? }\end{array}$ & $\begin{array}{l}\mathrm{Si} \\
12\end{array}$ & $\begin{array}{c}\text { No } \\
8\end{array}$ & 20 \\
\hline
\end{tabular}

36 Tecnologías de la información y la comunicación 


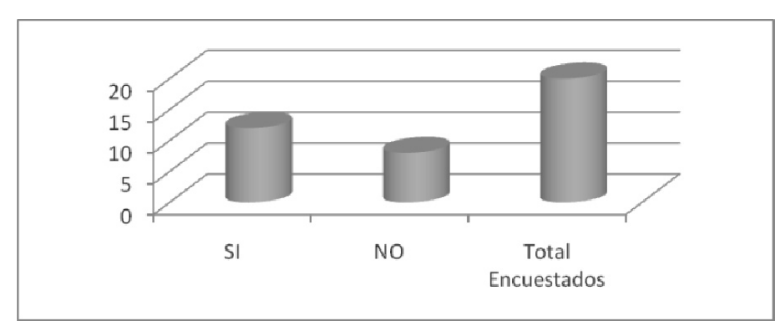

Figura 4. Resultado individual de la encuesta pregunta 2

Dado que el grupo de control seleccionado para el desarrollo investigativo es interdisciplinario y se encuentra en proceso formativo, se puede evidenciar en los resultados obtenidos, que el $60 \%$ de los estudiantes objeto de investigación han desarrollado trabajos colaborativos, es decir que en su proceso de formación han adelantado cursos por mediación virtual. Lo que les permite tener claridad acerca de la cooperación, contribución y construcción colectiva.

Tabla 4. Resultado individual de la encuesta, pregunta 3

\begin{tabular}{|l|c|c|c|c|}
\hline \multicolumn{2}{|c|}{ Pregunta } & \multicolumn{2}{l|}{ Prioridad en la dificultad } & Encuestados \\
\hline $\begin{array}{l}\text { De las siguientes opciones de posibles dificultades } \\
\text { encontradas en el desarrollo del trabajo de } \\
\text { investigación, haciendo uso del Software causa- } \\
\text { efecto, indique la prioridad de la dificultad } \\
\text { encontrada para cada una. }\end{array}$ & Alta & Media & Baja & \\
\hline $\begin{array}{l}\text { Dificultades para identificar el problema de } \\
\text { investigación }\end{array}$ & 2 & 7 & 11 & 20 \\
\hline Dificultad para diseñar el diagrama causa-efecto & 1 & 4 & 15 & 20 \\
\hline $\begin{array}{l}\text { Dificultad en el diseño en la construcción de los } \\
\text { instrumentos de recolección de la información }\end{array}$ & 2 & 3 & 15 & 20 \\
\hline $\begin{array}{l}\text { Dificultades para realizar el análisis de la } \\
\text { información recolectada }\end{array}$ & 2 & 4 & 14 & 20 \\
\hline
\end{tabular}

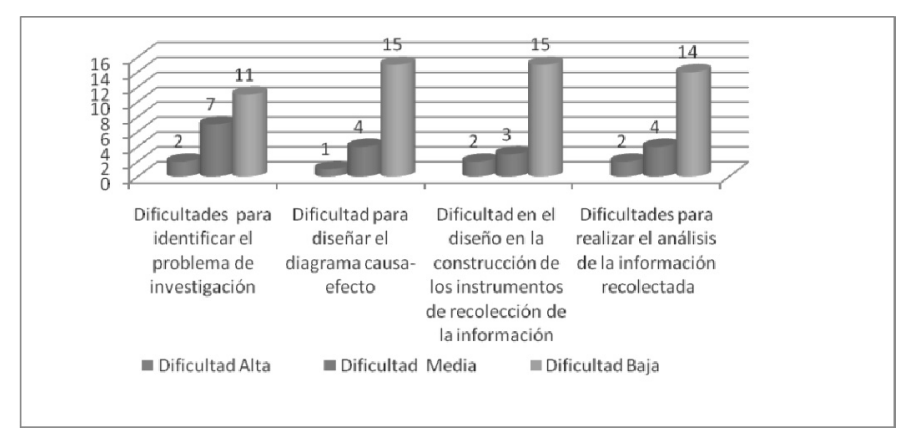

Figura 5. Resultado individual de la encuesta pregunta 3 
La aplicación del uso del software causa-efecto como herramienta de apoyo al proceso investigativo evaluado por el grupo de control, permite evidenciar según la figura 5, el grado de dificultad que obtuvieron los estudiantes en el desarrollo de su propuesta al proceso investigativo que adelantaron al interior del curso Metodología de investigación. Las posibles dificultades se enmarcaron en tres categorías: alta, media y baja.

Por otro lado, las cuatro premisas principales evaluadas en el proceso investigativo llevado a cabo por los estudiantes van orientadas a determinar el grado de dificultad en cada una. Para este caso, el de identificar el problema de investigación con el predominio del 55\% de dificultad baja. Mientras que el 35\% experimentó una dificultad media y sólo el 10\% de los encuestados manifestó una dificultad alta.

La segunda premisa hace referencia al diseño del diagrama causa-efecto, los resultados indican que la mayoría de los estudiantes objeto de control encontraron una baja dificultad correspondiente al $75 \%$, mientras que $20 \%$ experimentó una dificultad media y sólo el $5 \%$ indica haber tenido una alta dificultad.

La tercera premisa hace referencia a la dificultad en el diseño en la construcción de los instrumentos de recolección de la información, indicando que se tuvo una baja dificultad equivalente al $75 \%$, mientras que el 15\% indica que tuvo una dificultad media y sólo el 10\% experimentó una alta dificultad.

En la cuarta premisa se indaga acerca de las dificultades encontradas para realizar el análisis de la información recolectada, concluyendo que el 70\% manifestó haber obtenido una dificultad baja, mientras que el $20 \%$ indica que tuvo una dificultad media y sólo el 10\% experimentó una alta dificultad.

Tabla 5. Resultado individual de la encuesta, pregunta 4

\begin{tabular}{|l|c|c|c|}
\hline \multicolumn{1}{|c|}{ Pregunta } & \multicolumn{2}{|c|}{ Respuestas } & Encuestados \\
\hline ¿Considera usted que el software diseñado llamado & $\mathrm{Si}$ & No & \\
$\begin{array}{l}\text { Causa-Efecto, contribuye al desarrollo de trabajo } \\
\text { colaborativo y al aprendizaje cooperativo? }\end{array}$ & 17 & 3 & 20 \\
\hline
\end{tabular}

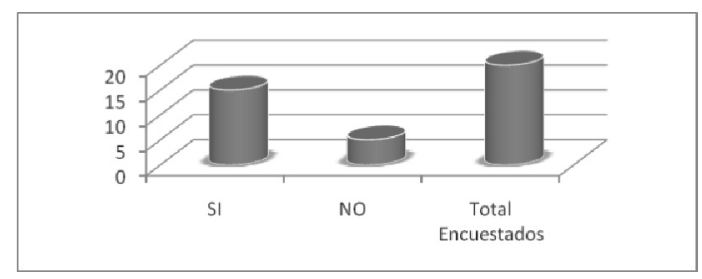

Figura 6. Resultado individual de la encuesta pregunta 4 
Los resultados obtenidos en la figura 6 correspondiente a la a la pregunta realizada indica que el $85 \%$ de los encuestados considera que el software causa-efecto, sí contribuye al desarrollo de trabajo colaborativo y al aprendizaje cooperativo, mientras que el 15\% manifiesta no estar de acuerdo.

Tabla 6. Resultado individual de la encuesta, pregunta 5

\begin{tabular}{|c|c|c|c|}
\hline Pregunta & \multicolumn{2}{|c|}{ Respuestas } & Encuestados \\
\hline ¿Considera usted que el software diseñado llamado & $\mathrm{Si}$ & No & \\
Causa-Efecto, contribuye al fortalecimiento de las & 15 & 5 & 20 \\
competencias investigativas en ambientes virtuales? & & & \\
\hline
\end{tabular}

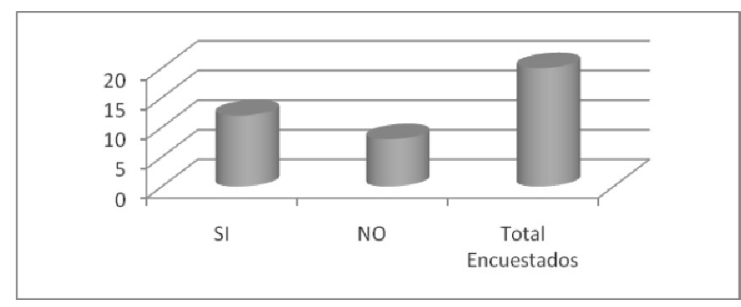

Figura 7. Resultado individual de la encuesta pregunta 5

De acuerdo con los resultados obtenidos en la figura 7, correspondiente a la pregunta realizada según la tabla 6 , indican que el $75 \%$ de los encuestados considera que el software causa-efecto, contribuye al fortalecimiento de las competencias investigativas en ambientes virtuales, mientras que el $25 \%$ manifiesta no estar de acuerdo con las competencias investigativas que puedan generarse con el uso del software.

Tabla 7. Resultado individual de la encuesta, pregunta 6

\begin{tabular}{|l|l|c|c|}
\hline \multicolumn{1}{|c|}{ Pregunta } & \multicolumn{2}{c|}{ Respuestas } & Encuestados \\
\hline ¿Considera usted que con el uso del software llamado & $\mathrm{Si}$ & No & \\
Causa-Efecto, cada estudiante es individualmente & 15 & 2 & 20 \\
responsable de manera equitativa del trabajo en grupo? & & & \\
\hline
\end{tabular}

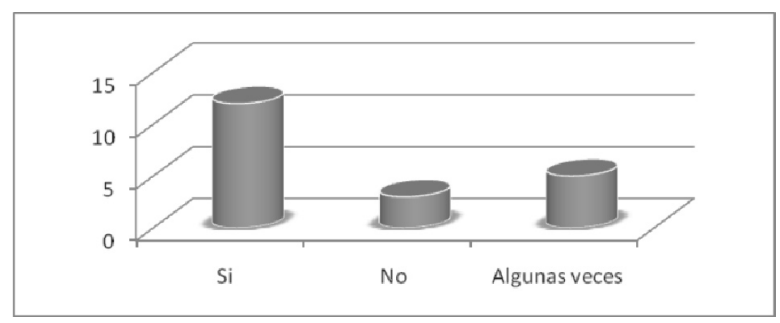

Figura 8. Resultado individual de la encuesta pregunta 6 
Una de las fortalezas del diagrama causa-efecto representado en el software es precisamente la capacidad para distribuir equitativamente el trabajo al interior del grupo, de tal manera que cada integrante es responsable de contribuir con su tarea encomendada, lo indica el 75\% tal como se puede evidenciar en los resultados obtenidos en la figura $\mathrm{n} .^{\circ} 8$, el $15 \%$ de los encuestados manifiestanque algunas veces se logra la equidad y la responsabilidad del trabajo en grupo.

Tabla 8. Resultado individual de la encuesta, pregunta 7

\begin{tabular}{|l|c|c|c|c|}
\hline \multicolumn{1}{|c|}{ Pregunta } & \multicolumn{3}{|c|}{ Respuestas } & Encuestados \\
\hline ¿Después de haber interactuado con el software & $\mathrm{Si}$ & No & Algunas veces & \\
Causa-Efecto, Considera usted que este es & 15 & 2 & 3 & 20 \\
intuitivo, amigable, de fácil manejo y permite & & & & \\
mayor interacción? & & & & \\
\hline
\end{tabular}

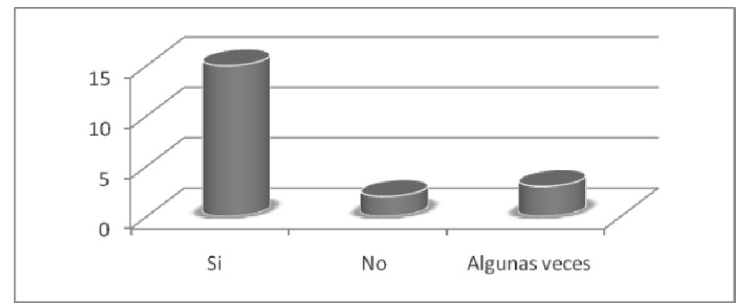

Figura 9. Resultado individual de la encuesta pregunta 7

Al indagar acerca de la facilidad de uso del software causa-efecto, por parte de los estudiantes que hicieron parte del grupo de control, se pudo determinar que el software es intuitivo, amigable y de fácil manejo, así lo indica el $75 \%$ de encuestados según los resultados obtenidos; por otro lado, el 15\% lo considera amigable e intuitivo sólo algunas veces; mientras que el 10\% manifiesta no ser intuitivo ni amigable.

\section{Análisis de resultados encontrados en la prueba de control}

Al finalizar el desarrollo de las actividades de los subgrupos, que se llevó a cabo en un periodo aproximado de sesenta (60) días de trabajo, se puede mencionar que la participación fue del 100\% de los estudiantes del curso seleccionado para el desarrollo de la actividad, es así como dentro de la autoevaluación realizada se evidenció que el consenso entre los participantes era su motivación por el desarrollo de la comunicación interna entre los miembros del grupo a través de herramientas telemáticas, fortaleciendo de esta manera el trabajo colaborativo, a la vez que los avances de las actividades individuales y de grupo quedaron registradas como evidencias para el seguimiento por parte de tutor del curso y del grupo investigador. 
Por otro lado, el grupo que trabajó de manera presencial pese a haber realizado la totalidad de las actividades propuestas, considera el trabajo adelantado de manera rutinaria, enfatizando en que se requirió la dedicación de tiempo, espacio y un lugar de encuentro entre los participantes para la socializar y consolidar el trabajo propuesto.

\section{CONCLUSIONES DE AVANCES PARCIALES DE LA INVESTIGACIÓN}

Después de haber realizado la validación del desarrollo tecnológico del software diagrama causa-efecto como pruebas de control dentro del ciclo de vida del desarrollo del Software, propuesta en la presente investigación con el grupo inicial de control, se consideró que técnicamente el aplicativo es amigable y estaba a punto para su buen uso.

En ese sentido se puede concluir que el uso del diagrama causa-efecto como instrumento de trabajo colaborativo en ambientes virtuales de aprendizaje contribuye a la formación de competencias investigativas acorde con el crecimiento global de las herramientas informáticas y telemáticas que fortalecen la comunicación en especial el uso de la internet como medio de interacción en la educación en línea e-Liaoning.

Teniendo en cuenta que dentro de la dinámica de la UNAD está el fortalecimiento de la metodología en la educación a distancia que viene evolucionando con el uso de las TIC's y la mediación virtual como medio de interacción al interior de los cursos académicos, se tiene que el $60 \%$ de los estudiantes objeto de investigación han desarrollado trabajos colaborativos, es decir, que en su proceso de formación han adelantado cursos por mediación virtual. Lo que les permite tener claridad y fortalecer su competencia de cooperación, contribución y construcción colectiva.

Así como se presentan algunas dificultades de baja proporción, en cuanto a la usabilidad y manejo del software causa- efecto, se evidencian fortalezas que apuntan a la capacidad para distribuir equitativamente el trabajo al interior del grupo, de tal manera que cada integrante es responsable de contribuir individual y colectivamente con su tarea encomendada, concluyendo que el software es intuitivo, amigable y de fácil manejo.

Si bien la herramienta que se construyó durante el desarrollo del proyecto se suma a la lista de software ya presentada, esta tiene la característica de ser flexible en su manejo, se adapta a la generación de competencias específicas esperadas, mientras que facilita el seguimiento y control en la participación de los estudiantes.

Asi mismo, se ha identificado en el grupo de estudiantes objeto de la investigación una motivación intrínseca por encontrar novedoso la forma de comunicación y la cooperación usando el software causa - efecto que garantiza la comunicación sincrónica por medio del chat y asincrónica en los espacios de interacción que permiten la publicación de los aportes individuales al interior de la estructura del software, como también permite al grupo debatir acerca de la identificación del problema, la construcción del instrumento para la recolección de los datos y genera espacios para el análisis de la información obtenida. 


\section{REFERENCIAS BIBLIOGRÁFICAS}

BRAVO, M., PIÑERO, M. (2008). Competencias docentes para nuevos entornos de Aprendizaje. Una propuesta curricular para la formación inicial y continua en la UPEL IPB. Edutec.

ESCOBEDO, H. (2001). Desarrollo de Competencias Básicas para Pensar Científicamente. Una propuesta didáctica para Ciencias Naturales. Bogotá. Colciencias.

GUITERT, M., ROMEU, T., PÉREZ, M. (2007). Competencias TIC y trabajo en equipo en entornos virtuales. Cataluña: Revista de Universidad y Sociedad del Conocimiento. Universitat Oberta de Catalunya.

MALDONADO, L., LANDAZÁBAL, D., HERNÁNDEZ, J., RUIZ, Y., CLARO, A., VANEGAS, H., CRUZ, S. (2007). Visibilidad y Formación en Investigación. Estrategias para el desarrollo de competencias investigativas. Bogotá, Colombia: Universidad Nacional Abierta y a Distancia UNAD.

MUÑOZ, J., QUINTERO, J., MUNEVAR, R. Como desarrollar competencias investigativas en la educación. Consultado en Marzo, 28, de 2009 en http://books.google.com.co/bo oksid2kxYbr8bdkIC\&dqcompetenciasinvestigativas\&printsecfrontcover\&sourcebl\&ots 4OtS5zplED\&sigr4BwPXpO54iX2u7o_RUJpc2kYgs\&hles\&ei4dPgSZTyJqKHmQfpsS0Dw\&saX\&oibook_result\&ct result\&resnum8PPP1,M2.

PINZÓN, Y. Y OTROS. Las TIC's como instrumento de mediación pedagógica en el aprendizaje colaborativo: Software para el diseño de Diagramas CAUSA-EFECTO. Tesis de grado para obtener el título de Ingeniero de Sistema. UNAD, CEAD Popayán. 2006.

RODRÍGUEZ, M., GARCÍA, M. (2000). Formación reflexiva - creativa de competencias Investigativas en los docentes. 